\title{
Chronic suppurative otitis media due to Streptomyces cacaoi, the second case report in human infection
}

\author{
Lu Ai, Han Huang, Zhongwen Wu, Pingjuan Liu, Jianyu Huang and Yili Chen*
}

\begin{abstract}
Background: Streptomyces cacaoi, Gram-positive, branched, filamentous bacillus forms without fragmentation, are saprophytic soil organisms rarely known to cause invasive infections other than mycetoma. Here we describe a case of chronic suppurative otitis media caused by Streptomyces cacaoi in a patient with hyperlipidemia in China.

Case presentation: A 62-year-old female patient with hyperlipidemia suffered chronic suppurative otitis media caused by Streptomyces cacaoi. She had a favorable outcome with a 4-week course of ofloxacin ear drops.

Conclusions: Streptomyces cacaoi is rarely reported to cause human infection. The introduction of molecular techniques improves the ability to identify rare species such as Streptomyces considerably. We report the case improve our ability to identify this pathogen and expand the range of known bacterial causes of human infection.
\end{abstract}

Keywords: Streptomyces cacaoi, Chronic suppurative otitis media, Case report

\section{Background}

Streptomyces cacaoi, classified among the aerobic actinomycetes, presented Gram-positive, branched, filamentous bacillus forms without fragmentation with optimum growth at $35^{\circ} \mathrm{C}$ [1]. Streptomyces are saprophytic soil organisms rarely known to cause invasive infections other than mycetoma. Ubiquitous nature and the low pathogenicity of Streptomyces organisms make most clinical isolates contaminants or colonizers [2, 3]. Until 2012 Gerald J. Pellegrini et al. first reported a scalp infection patient from Pondicherry, India and 16S rRNA sequence analysis provided the species identification [4]. Here we describe a case of chronic suppurative otitis media caused by Streptomyces cacaoi in a patient with hyperlipidemia in China, tending to improve our capacity to identify this isolate and widen the field of known bacterial causes of human infection.

\footnotetext{
* Correspondence: 845475702@qq.com

Department of Laboratory Medicine, The First Affiliated Hospital of Sun Yat-sen University, Guangzhou 510080, Guangdong, China
}

\section{Case presentation}

A 62-year-old woman presented to medical care (The First Affiliated Hospital of Sun Yat-sen University, Guangzhou, China) due to repeated purulence and decreased hearing of right ear for 30 years, and aggravated for 1 year. The accidentally insect flew into the right ear, and then purulence and decreased hearing developed and sustained for 30 years. The patient had not been treated with antibiotics for ear infection before. Besides, she had a history of hyperlipidemia and treated with traditional Chinese Medicine.

Upon medical checkup, she had a tympanic temperature of $36.5^{\circ} \mathrm{C}$, blood pressure of $129 / 87 \mathrm{mmHg}$, pulse rate of $72 / \mathrm{min}$ and respiratory rate of $19 / \mathrm{min}$. Computed tomography of mastoid process revealed chronic mastoiditis and tympanic membrane perforation of right side. Aural endoscopy showed that the left external auditory canal unobstructed and the tympanic membrane intact with local calcified plaques; white aerial hyphae can be seen in the right external auditory canal, with large perforation in pars tensa of tympanic membrane, and drum room clean 

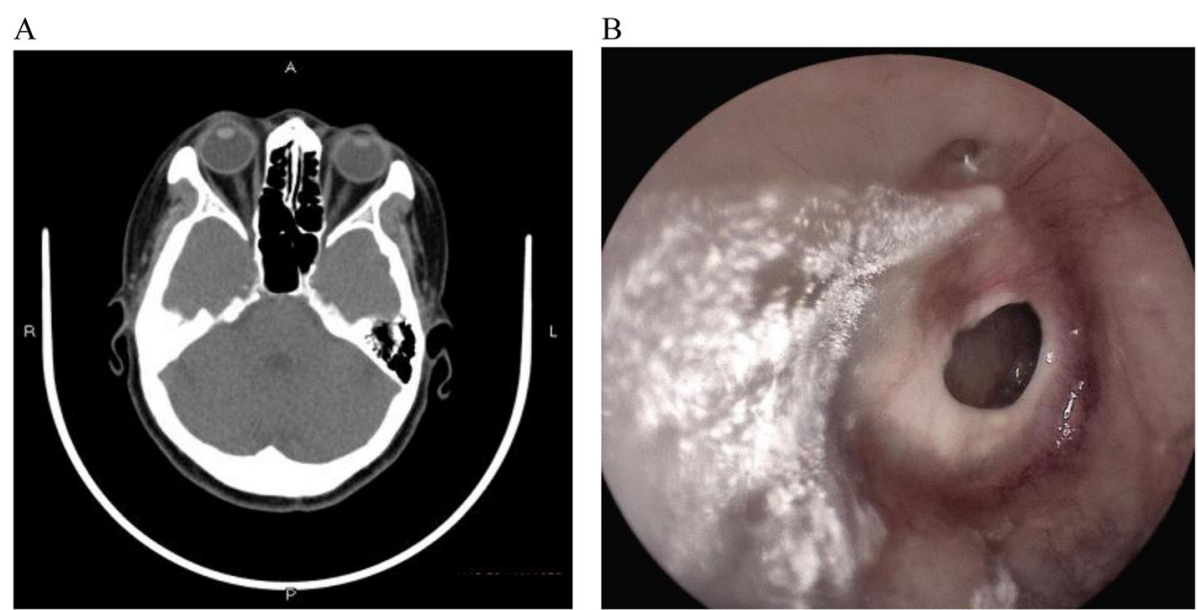

Fig. 1 Computed tomography of mastoid process revealed chronic mastoiditis and tympanic membrane perforation of right side (a), and white aerial hyphae can be seen in the right external auditory canal, with large perforation in pars tensa of tympanic membrane in aural endoscopy (b)

(Fig. 1). Laboratory evaluation revealed leukocyte count of $6810 / \mathrm{mm}^{3}$ with $68.9 \%$ neutrophils and $23.7 \%$ band forms, total cholesterol $7.2 \mathrm{mmol} / \mathrm{L}$ (normal range, 3.1-5.7 $\mathrm{mmol} / \mathrm{L}$ ), high-density lipoprotein $2.02 \mathrm{mmol} / \mathrm{L}$ (normal range, $1.09-1.63 \mathrm{mmol} / \mathrm{L}$ ), lipoprotein(a) $860 \mathrm{mg} / \mathrm{L}$ (normal range, $60-300 \mathrm{mg} / \mathrm{L}$ ).

On day 4, the patient underwent microscope supporting right ear tympanoplasty, ossicular chain release and aticoantrotomy under general anesthesia. Empiric antibiotic treatment with Cefuroxime sodium $(1.5 \mathrm{~g}$ every day) were started and lasted 1 day for prevention of postoperative infection. Ear exudate intraoperative was collected and the Gram stain of the colonies demonstrated Gram-positive branched filamentous bacilli, with weak acid fast staining negative (Fig. 2a). The organism appeared grayish white, dry, wrinkled small colonies biting agar after $24 \mathrm{~h}$ incubation on blood agar, beta hemolysis obvious after $48 \mathrm{~h}$ incubation. The isolate exhibited distinctive powder or velvet colonies that developed characteristic white aerial hyphae after $72 \mathrm{~h}$ of

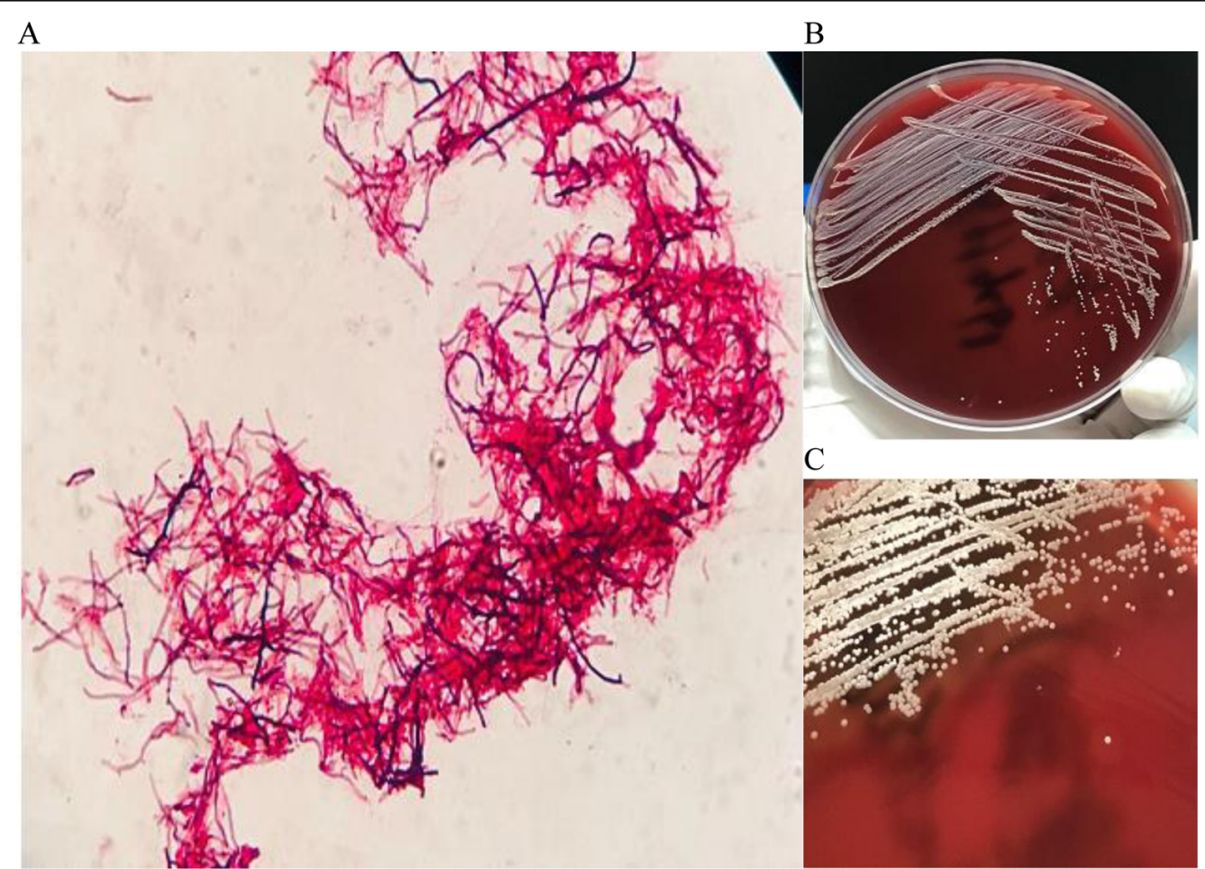

Fig. 2 Gram stain of ear exudate, demonstrating Gram-positive, filamentous, non-spore-forming bacilli (a), and The organism appeared grayish white, dry, wrinkled small colonies biting agar after $24 \mathrm{~h}$ incubation on blood agar (b), beta hemolysis obvious after $48 \mathrm{~h}$ incubation (c) 
aerobic growth (Fig. 2b, c). To confirm the identity of the isolate, we performed molecular identification using primer sets 16S-forward (5'AGAGTTTGATCCTGGCTCAG3') and 16S-reverse (5'GGTTACCTTGTTACGACTT3') by polymerase chain reaction amplification of the 16S rRNA gene. The sequence analysis of 1,450 bp nucleotide were queried against the GenBank $16 \mathrm{~S}$ rRNA gene database and the best match returned was the Streptomyces cacaoi (GenBank accession number AB184115), with $99.2 \%$ similarity.

Due to the limitations of experimental conditions, the antibiotic susceptibility of the Streptomyces cacaoi strain was mostly determined by the Kirby-Bauer disk diffusion method on Mueller-Hinton agar plates. Although there are no categorical interpretative criteria for antimicrobial susceptibility testing data for Streptomyces cacaoi, the isolate inhibition zone (millimeter) for antimicrobials demonstrated: minocycline $20 \mathrm{~mm}$, gentamicin $37 \mathrm{~mm}$, clindamycin $6 \mathrm{~mm}$, erythromycin $6 \mathrm{~mm}$, sulphamethoxazole/trimethoprim $6 \mathrm{~mm}$, amikacin $32 \mathrm{~mm}$, levofloxacin $6 \mathrm{~mm}$, moxifloxacin $20 \mathrm{~mm}$, ciprofloxacin $12 \mathrm{~mm}$, linezolid $50 \mathrm{~mm}$, cefotaxime $6 \mathrm{~mm}$, ceftriaxone $6 \mathrm{~mm}$, cefepime $6 \mathrm{~mm}$. Moreover MIC testing indicated resistance to amoxicillin-clavulanic acid $(>256 \mu \mathrm{g} / \mathrm{ml})$, meropenem $(>32 \mu \mathrm{g} / \mathrm{ml})$, and intermediate resistance observed to imipenem $(6 \mu \mathrm{g} / \mathrm{ml})$ with the employ of Etest (Biomerieux SA, Marcy, France).

With external application of ofloxacin ear drops sustained for 2 weeks after surgery, the patient returned for pure tone audiometry and ear endoscopy and recovered in good condition. One month later she accepted cleaning of the right external auditory canal which was packed with ear dressing under the aural endoscope and was delighted to see the intact tympanic membrane after operation (Fig. 3).

\section{Discussion and conclusions}

The Streptomyces cacaoi, which was discovered and isolated from cacao beans, was primitively described by Waksman in 1932, corrected by Waksman and Henrici in 1948 [5], and at last revised by Lanoot et al. as S. Cacaoi in 2002 [6]. The organism, classified among the aerobic actinomycetes, presented Gram-positive, branched, filamentous bacillus forms without fragmentation with optimum growth at $35^{\circ} \mathrm{C}$. The organism stained acid fast negative by the modified Kinyoun method, which is different from other members of the group, such as Nocardia spp. And Rhodococcus spp..

The Streptomycetes are classified as a separate genus within the aerobic actinomycetes, most well known for the approximately 600 different species [3]. Ubiquitous nature and the low pathogenicity of Streptomyces organisms make most clinical isolates pollutants or colonizers $[7,8]$. For it is difficult to determine the pathogenicity of the omnipresent Streptomyces definitively, several guiding principles have been given to assist the diagnosis of actual infection: isolate from sterile site, morphological identification under direct microscope, and eliminate other pathogeny by Kapadia et al. [9]. A stringent clinical and microbiologic correlation is needed in this regard, such as clinical manifestations, isolation of the organism from sterile sources (ideally in large quantities), direct microscopic identification in infected tissue [10]. Direct specimen microscopy is extremely important, and many times extended incubation time can prove effective [6]. In this case, white aerial hyphae observed by aural endoscopy initially made us confused whether it was fungus otitis media or suppurative otitis media, but the morphology observed by the microscope gave some effective clues. Though the morphology changed with the strains and their growth stages in infected tissues, bacterial culture remains the gold standard for final etiological diagnosis to date. On culturing, Streptomyces cacaoi produces dry, chalky, gray-white colonies that emit pungent, musty odor or earthy scent [11].

In the last decade, owing to the extensive use of PCR and DNA sequencing identification to the species level has evolved to more polyphasic approach, in the meantime $16 \mathrm{~S}$ rDNA sequencing has played a critical role in more accurate identification of bacterial isolates and the discovery of novel bacteria in clinical microbiology laboratories [12]. For the identification of bacteria with
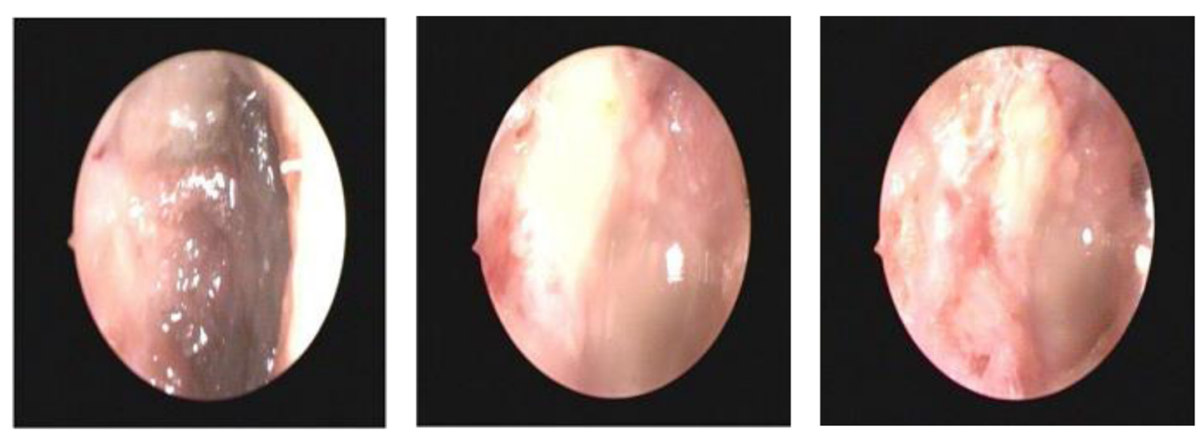

Fig. 3 The aural endoscope indicated intact tympanic membrane after operation 
unusual phenotypic profiles, emergent bacteria, slow growth bacteria, uncultivable bacteria and culturenegative infections, $16 \mathrm{~S}$ rDNA sequencing make a big difference [11]. We can reasonably speculate that previously similar Streptomyces infections were under diagnosed on account of technical and methodological limitations, the lack of molecular techniques included.

The susceptibility of the Streptomyces cacaoi to different antimicrobials was performed according to Clinical and Laboratory Standards Institute guidelines M24-A2 using broth micro dilution method [13]. The currently recommended experience therapy for Streptomyces actinomycetoma infection is trimethoprim-sulfamethoxazole which is used worldwide. But in an extensive investigate of 92 isolates by Rose et al., the susceptibility testing results stated a critical degree of resistance to many antibiotics: $79 \%$ of strains were resistant to ceftriaxone, $75 \%$ to sulfamethoxazole and to erythromycin, $65 \%$ to trimethoprim-sulfamethoxazole, $54 \%$ to ciprofloxacin, and all were sensitive to amikacin and linezolid [14]. It suggested that the fusidic acid, amikacin, novobiocin, doxycycline, gentamicin, and linezolid combination with trimethoprim-sulfamethoxazole should be reconsidered. Meanwhile the fact that Streptomyces spp. are slowgrowing bacteria renders prolongation of antibacterial treatment necessary [15].

To our knowledge, this is the second report of Streptomyces cacaoi human infection. As the experience limited, we need further research to better understand the isolates of the genus Streptomyces: the predisposing factors for infection, and the process, treatment, and evolution of these infections. On the other hand, the introduction of molecular techniques applied to a bacterial isolate has brought about a vast improvement in the section of identify rare species, especially in a taxon such as Streptomyces, which consists of approximately 600 different species.

\section{Abbreviations}

S. Cacaoi: Streptomyces Cacaoi; PCR: Polymerase Chain Reaction;

DNA: Deoxyribonucleic Acid; rDNA: ribosome Deoxyribonucleic Acid

\section{Acknowledgements}

None.

\section{Authors'contributions}

YLC traced the case, was responsible for identifying the bacteria, and was a major contributor in writing the manuscript. LA participated in the design and analyzing the sequence. $\mathrm{HH}, \mathrm{ZWW}, \mathrm{PJL}$ and JYH participated in strain collection and literature searching. All authors read and approved the final manuscript.

\section{Funding}

None.

\section{Availability of data and materials}

Data and materials of this report are publicly available from the corresponding author on reasonable request.

\section{Ethics approval and consent to participate}

This report was approved by the Clinical Research and Ethics Committee of the First Affiliated Hospital of Sun Yat-sen University.

\section{Consent for publication}

Written informed consent for publication of the clinical details including the medical history, bacteria cultures, pictures, videos and text was obtained from the patient.

\section{Competing interests}

The authors declare that they have no competing interests.

Received: 9 February 2020 Accepted: 2 July 2020

Published online: 11 July 2020

\section{References}

1. Conville PS, Witebsky FG. Nocardia, Rhodococcus, Gordonia, Actinomadura, Streptomyces, and other aerobic actinomycetes, p 515-542. In: Murray PR, Baron EJ, Jorgensen JH, Landry ML, Pfaller MA, editors. Manual of clinical microbiology. 9th ed. Washington, DC: ASM Press; 2007.

2. Manteca A, Pelaez Al, del Mar Garcia-Suarez M, et al. A rare case of silicone mammary implant infection by Streptomyces spp. in a patient with breast reconstruction after mastectomy: taxonomic characterization using molecular techniques. Diagn Microbiol Infect Dis. 2009;63:390-3.

3. McNeil MM, Brown JM. The medically important aerobic actinomycetes: epidemiology and microbiology. Clin Microbiol Rev. 1994;7:357-417.

4. Pellegrini GJ Jr, Graziano JC, Ragunathan L, et al. Scalp Abscess Due to Streptomyces cacaoi subsp.cacaoi, First Report in a Human Infection. J Clin Microbiol:1484-6.

5. Waksman S, Henrici A. The nomenclature and classification of the actinomycetes. J Bacteriol. 1943;46:337-41.

6. Lanoot B, et al. The search for synonyms among streptomycetes by using SDS-PAGE of whole cell proteins. Emendation of the species Streptomyces aurantiacus, Streptomyces cacaoi subsp.cacaoi, Streptomyces caeruleus and Streptomyces violaceus. Int J Syst Evol Microbiol. 2002;52(Pt. 3):823-9.

7. Chander J, Singla N, Handa U. Human cervicofacial mycetoma caused by Streptomyces griseus: First case report. J Microbiol Immunol Infect. 2015;48: 703-5.

8. Ariza-Prota MA, Pando-Sandoval A, Fole-Vázquez D, et al. Communityacquired bacteremic Streptomyces atratuspneumonia in animmunocompetent adult: a case report. J Med Case Rep. 2015;9:262. https://doi.org/10.1186/s13256-015-0753-y.

9. Kapadia M, Rolston KVI, Han XY. Invasive Streptomyces infections. Am J Clin Pathol. 2007:127:619-24.

10. Kofteridis DP, Maraki S, Scoulica E, et al. Streptomyces pneumonia in an immunocompetent patient: a case report and literature review. Diagn Microbiol Infect Dis. 59:459-62.

11. Woo PCY, Lau SKP, Teng JLL. Then and now: use of $16 \mathrm{~S}$ rDNA gene sequencing for bacterial identification and discovery of novel bacteria in clinical microbiology Laboratories. Clin Microbiol Infect. 2008;14:908-34.

12. Shao S, Guo X, Guo P, et al. Roseomonas mucosa infective endocarditis in patient with systemic lupus erythematosus: case report and review of literature. BMC Infect Dis. 2019;19:140

13. CLSI/NCCLS. Susceptibility testing of mycobacteria, nocardiae, and other aerobic actinomycetes. Approved standard M24-A. Wayne: National Committee for Clinical Laboratory Standards; 2003.

14. Rose CE III, Brown JM, Fisher JF. Brain abscess caused by Streptomyces infection following penetration trauma: case report and results of susceptibility analysis of 92 isolates of Streptomyces species submitted to the CDC from 2000 to 2004. J Clin Microbiol. 2008;46:821-3.

15. Hamid ME. Variable antibiotic susceptibility patterns among Streptomycesspecies causing actinomycetoma in man and animals. Ann Clin Microbiol Antimicrob. 2011;10:24 doi:1186/1476-0711-10-24 (formerly doi: 10.1073/pnas.041593698).

\section{Publisher's Note}

Springer Nature remains neutral with regard to jurisdictional claims in published maps and institutional affiliations. 